\title{
S-15-5 Chemical Spectroscopic and Crystallographic Studies of UDP-Galactose 4-Epimerase from Escherichia coli
}

\author{
P.A.Frey, A.J.BAuer, J.L.VAnhoOKe, J.M.KonopKA, \\ I.RAYMENT, and H.M.HOLDEN
}

Institute for Enzyme Research and the Departments of Biochemistry and Chemistry, University of Wisconsin-Madison, Madison, Wisconsin 53705, U. S. A.

\section{INTRODUCTION}

UDP-galactose 4-epimerase catalyzes an essential reaction in the metabolism of galactose, the interconversion of UDP-galactose with UDP-glucose. This is part of the Leloir pathway of galactose metabolism, a portion of which is shown in the following scheme for the conversion of galactose into

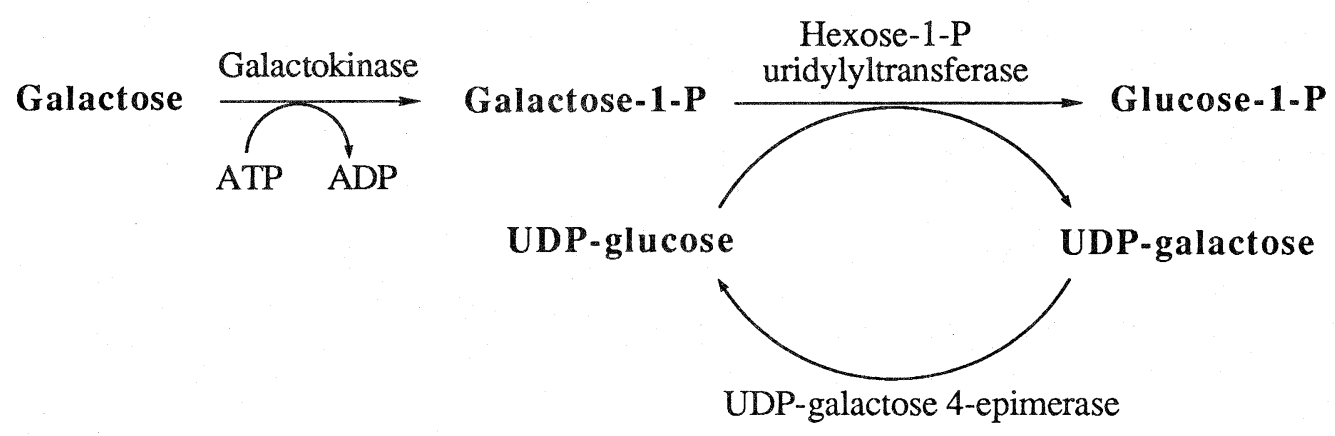

glucose-1-P. This process is catalyzed by galactokinase, hexose-1-P uridylyltransferase, and UDPgalactose 4-epimerase, which are encoded in $E$. coli by the gal operon.

UDP-galactose 4-epimerase from $E$. coli, originally described by D. M. Wilson and D. S. Hogness, is a dimer of identical subunits with an overall molecular weight of 79,000. The molecular properties and mechanistic studies of the epimerases from $E$. coli and yeast have recently been reviewed [1]. The purified enzyme contains NAD+, which is transiently and reversibly reduced by substrates to the catalytic intermediate $\mathbf{E} \cdot \mathrm{NADH} \cdot \mathrm{UDP}-4$-ketoGlc as follows:

$$
\text { E.NAD }{ }^{+}+\text {UDP-Gal }
$$$$
\text { if }
$$

\section{E.NAD + UDP-Gal}

$$
\mathbf{E} \cdot \mathrm{NAD}^{+}+\mathrm{UDP}-\mathrm{Glc}
$$

$$
\text { 11 }
$$

\section{$\mathrm{E} \cdot \mathrm{NAD}+\mathrm{UDP}-\mathrm{GlC}$}

Nonstereospecific hydrogen transfer between NAD+ and UDP-hexoses at the active site is thought to arise from conformational mobility of the 4-ketoglucosyl moiety within the complex E.NADH.UDP4-ketoGlc. This hypothesis is supported by studies showing strong binding between the active site and the nucleotide moieties of substrates and very weak interactions with the sugar moieties. The active site binds sugars weakly and with little specificity.

The binding energy made available by the interaction of epimerase with the nucleotide moieties of substrates is used to catalyze hydride transfer between the glycosyl moieties and the pyridine nucleotide. This effect is most clearly revealed by the interaction of UMP with this enzyme. UMP is 
a competitive inhibitor which, upon binding to the active site, greatly enhances the chemical reactivity of NAD+. The complex E.NAD+.UMP is rapidly reduced to E.NADH.UMP by reducing sugars, $\mathrm{NaBH}_{3} \mathrm{CN}$, and even Tris buffer. The resting enzyme (E.NAD+) does not react at a detectable rate with any of these reducing agents. UDP-benzene and the dianionic form of UDP also enhance the reducibility of $\mathrm{NAD}+$ associated with this enzyme.

\section{P-31 NMR SPECTRA OF EPIMERASE-NUCLEOTIDE COMPLEXES}

The simplest means by which uridine nucleotides could increase the chemical reactivity of NAD+ at the active site of the epimerase is to induce a conformational change in the enzyme that has the effect of increasing the chemical reactivity of NAD+ through enzyme binding interactions. Such an effect is revealed in the P-31 NMR spectra of NAD+ in the epimerase and epimerase-UMP complex [2]. The P-31 chemical shifts in Table 1 show that the binding of UMP changes both of the P-32

TABLE 1

P-31 Chemical shifts for NAD+ and NADH in complexes of UDP-galactose 4-epimerase

$\begin{array}{lll}\text { E.NAD } & \text { E.NAD.UMP } & \text { E.NADH.UMP }\end{array}$
$\delta_{\mathrm{P}}(\mathrm{ppm})$
$-9.60,-12.15$
$-10.51,-11.06$
$-10.57$

chemical shifts of NAD+ by more than $1 \mathrm{ppm}$ upon binding to the active site. Upon reduction with glucose, the two signals coalesce into an unresolved signal. All spectra of the E.NAD+ also reveal a small, poorly resolved signal $(-10.5 \mathrm{ppm})$ between the two major signals that is thought to represent NADH associated with the purified enzyme. The simplest rationale for the difference in P-31 chemical shifts for NAD+ in Table 1 is that UMP induces a protein conformational change that alters the binding interactions between the enzyme and NAD+. However, the NMR data alone cannot exclude the possibility that a direct interaction between UMP and NAD + at the active site could cause the perturbations.

\section{CRYSTAL STRUCTURE OF UDP-GALACTOSE 4-EPIMERSASE}

UDP-galactose 4-epimerase from $E$. coli cocrystallizes with the competitive inhibitor UDPbenzene in a form that is suitable for three-dimensional structural analysis by $x$-ray diffraction [3]. The crystals belong to the orthorhombic space group P $2{ }_{1} 2_{1} 2_{1}$, and the dimensions of the unit cell are $a=76.3 \AA, b=83.1 \AA$, and $c=132.1 \AA$. The crystals diffract to a resolution of better than $2.3 \AA$. The unit cell contains a single dimer of UDP-galactose 4-epimerase complexed with UDP-benzene.

The structure of the epimerase-UDP-benzene complex has been solved at a resolution of $2.5 \AA$

[4]. The $\alpha$-carbon chain for one subunit is shown in Figure 1, with the structures of NAD+ and UDP-benzene shown in their respective binding sites. The structure reveals many important facts, some of which dramatically alter thought about this enzyme. The subunits of the dimer are crystallographically identical at $2.5 \AA$ resolution and related by a two-fold axis of symmetry. The chain fold in the large $\mathrm{N}$-terminal domain is very similar to that in pyridine nucleotide-dependent dehydrogenases; it consists of the seven-stranded twisted $\beta$-sheet surrounded by $\alpha$-helices that characterizes the dinucleotide fold. The C-terminal 120 amino acids are folded into a smaller domain, and the cleft between the two domains forms the binding site for substrates and NAD + . The structure clearly shows that UDP-benzene does not interact directly with the pyrophosphoryl moiety of NAD + . It is, therefore, unlikely that a direct interaction between UMP and NAD+ would perturb the phosphorus chemical shifts of NAD +. The effect of UMP on the phosphorus chemical shifts and chemical reactivity of NAD + is probably mediated through a protein conformational change. The structure of the epimerase-UDP-benzene complex in Figure 1 is probably that of the activated conformation of the protein.

UDP-galactose 4-epimerase, in contrast to enzymes such as lactate dehydrogenase, binds NAD+ essentially irreversibly. The structure at $2.5 \AA$ resolution allows us to consider what might be the physicochemical basis for the strong binding of $\mathrm{NAD}^{+}$at the active site. The close contacts between 

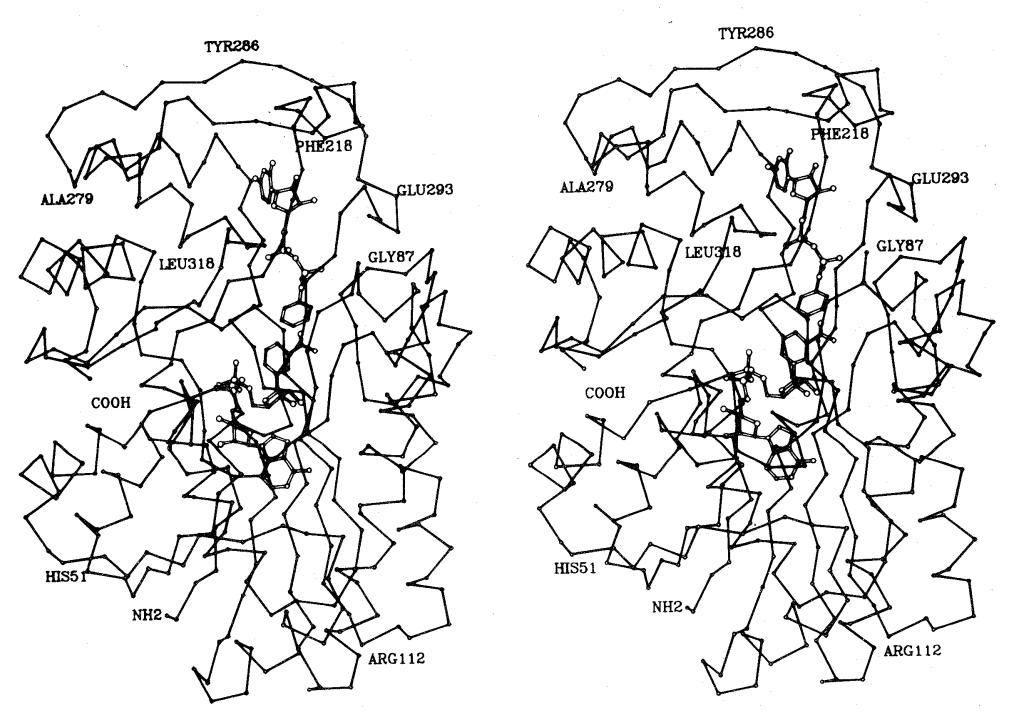

Fig. 1 A stereoview of the $\alpha$-carbon chain for one subunit of UDP-galactose 4-epimerase.

$\mathrm{NAD}+$ and its binding site are numerous, especially around the nicotinamide ribosyl and the adenosyl moieties. The close contacts in the $2.5 \AA$ resolution structure are listed in Table 2 . The contacts between the adenosyl moiety and the protein constitute a hydrogen bonded network that essentially cross links the adenosyl moiety to the binding site. There are many more hydrogen bonds linking the nicotinamide ribosyl and adenosyl groups to the protein than there are in typical pyridine nucleotide dehydrogenases such as lactate dehydrogenase.

TABLE 2

Potential H-bonded protein-NAD+ interactions in UDP-galactose 4-epimerase

Nicotinamide ribosyl
carboxamide-Phe $178(\mathrm{NH})$
carboxamide-Ser 124-OH
carboxamide-Tyr 149-OH
2'-OH-Tyr 149-OH
2', 3'-OH-Lys $153-\varepsilon-\mathrm{NH}_{3}{ }^{+}$
3'-OH-Phe $80(\mathrm{C}=\mathrm{O})$

Pyrophosphate

P1-Lys $84-\varepsilon-\mathrm{NH}_{3}+$

P1-Tyr $11(\mathrm{NH})$

P2-Tyr $11(\mathrm{NH})$

$\mathrm{P} 2-\mathrm{Asn} 35-\mathrm{CONH}_{2}$

$\frac{\text { Adenosyl }}{\text { N6-Asn 99(CONH} 2)}$
N1-Ile $(\mathrm{NH})$
N3-Asn 32 $(\mathrm{NH})$
N7-Asn $32(\mathrm{CONH})$
2'-OH-Asp 32 $(\mathrm{COO})$
3'-OH-Ser 36 $(\mathrm{NH}, \mathrm{C}=\mathrm{O})$
3'-OH-Asn 35 $(\mathrm{C}=\mathrm{O})$

\section{THE PYRIDINE NUCLEOTIDE CONTENT OF UDP-GALACTOSE 4-EPIMERASE}

Wilson and Hogness originally reported that UDP-galactose 4-epimerase from $E$. coli contains one molecule of NAD+ per dimer of identical subunits, based on enzymatic assays of NAD+ released upon denaturation of the enzyme with perchloric acid [5]. In studies conducted in this laboratory, the NAD + content reported by Wilson and Hogness has been confirmed; moreover, upon UMPdependent reductive inactivation with glucose, spectrophotometric measurements at $345 \mathrm{~nm}$ indicated the formation of one NADH for each pair of subunits.

Nevertheless, the structure clearly shows the presence of one pyridine nucleotide and one UDPbenzene bound to each subunit of the crystalline enzyme dimer. All questions that had been raised by the apparent presence of one $\mathrm{NAD}^{+}$and one active site per dimer are cast into a new light by this fact. The $\mathrm{NAD}^{+}$and active site are not at the interface of subunits. The structure does not exemplify a case of extreme anticooperativity, in which the binding of NAD+ at the active site in one subunit induces conformational changes that prevent the other subunit from binding a pyridine nucleotide. 
The presence of two pyridine nucleotides per dimer is further supported by analyses of enzyme samples for total phosphorus and for total organic phosphate. The data are presented in Table 2, and are consistent with the presence of four moles of organic phosphate per mole of dimeric enzyme.

TABLE 3

Phosphorus and organic phosphate content of UDP-galactose 4-epimerase

\begin{tabular}{lccccc}
\hline & Analysis for S/P $a$ & & \multicolumn{2}{c}{ Analysis for organic phosphate ${ }^{b}$} \\
\cline { 2 - 3 } Epimerase & S/P & & P/dimer & Epimerase & Org. phosphate/dimer \\
$0.054 \mu \mathrm{mol} / \mathrm{L}$ & 4.2 & $5.7 c$ & $0.70 \mathrm{nmol}$ & $4.1 \pm 0.2$ \\
$0.107 \mu \mathrm{mol} / \mathrm{L}$ & 4.6 & $5.2 c$ & $1.05 \mathrm{nmol}$ & $4.1 \pm 0.1$ \\
\hline
\end{tabular}

$a$ Analysis for sulfur and phosphorus was by inductively coupled plasma emission spectroscopy.

$b$ Epimerase was measured by $\mathrm{A} 280$ and by $\triangle \mathrm{A} 345$ upon UMP-dependent reduction by glucose. Organic phosphate was measured as inorganic phosphate in ashed samples.

$c$ Calculated based on 24 sulfur-containing amino acids per dimer.

The analysis of phosphorus by inductively coupled plasma emission spectroscopy was based on the ratio of sulfur to phosphorus in a given sample. The values obtained depended to a considerable extent on the amount of $P$ and $S$ in the buffers, which were below the detectable range. Given the maximal detectable $\mathrm{P}$ and $\mathrm{S}$ in the background, the calculated $\mathrm{P} /$ dimer content could range from 4.7 to 5.9. In the analysis for organic phosphate, the enzyme samples were ashed and assayed for $P_{i}$. The unashed samples contained no detectable $\mathrm{P}_{\mathrm{i}}$, so that the analysis of ashed samples corresponded to organic phosphorus. The calculation of phosphate per dimer depended on the spectrophotometric measurement of enzyme concentration. The two analyses show that the enzyme contains at least four gram atoms of $\mathrm{P}$ per mole of dimeric protein.

The pyridine nucleotide in one of the subunits of dimeric UDP-galactose 4-epimerase is NAD+. The other subunit also contains a nucleotide that appears to be a pyridine nucleotide but does not appear to be NAD+.

\section{ACKNOWLEDEMENTS}

This research was supported by grants GM 30480 to PAF and GM 39082 to HMH from the National Institute of General Medical Science of the USPHS.

\section{REFERENCES}

[1] Frey, P.A. (1987): "Complex Pyridine Nucleotide-Dependent Transformations", in Pyridine Nucleotide Coenzymes: Chemical, Biochemical, and Medical Aspects, ed. by Dolphin, D., Poulson, R., and Avramovic, O.,Vol. 2B, Wiley \& Sons, New York, pp. 461-511.

[2] Konopka, J.M., Halkides, C.J., Vanhooke, J.L., Gorenstein, D.G., and Frey, P.A. (1989): UDP-galactose 4-epimerase. P-31 Nuclear Magnetic Resonance of NAD+ and NADH Bound at the Active Site. Biochemistry 28, 2645-2654.

[3] Bauer, A. J., Rayment, I., Frey, P. A., and Holden, H. M. (1991): The Isolation, Purification, and Preliminary Crystallographic Characterization of UDP-galactose 4-Epimerase from Escherichia coli. Proteins: Structure, Function and Genetics 9, 135-142.

[4] Bauer, A. J., Rayment, I., Frey, P. A., and Holden, H. M. The Molecular Structure of UDPGalactose 4-Epimerase from Escherichia coli at $2.5 \AA$ Resolution. Proteins: Structure, Function, and Genetics, In press.

[5] Wikson, D. B., and Hogness, D. S. (1964): The enzymes of the galactose operon in Escherichia coli: Purification and characterization of uridine diphosphate galactose 4epimerase. J. Biol. Chem. 239, 2469-2476. 